\title{
THROMBOSIS OF THE INFERIOR VENA \\ Case Report
}

\author{
By M. J. GreenberG, M.B., M.R.C.P. \\ and D. McC. GRegG, D.M.R., D.M.R.D. \\ Addenbrooke's Hospital, Cambridge
}

Inferior vena caval obstruction occurs not infrequently. In I9I I Pleasants reviewed 314 published cases of which 68 per cent. were due to thrombosis, I 6 per cent. to external pressure and I 6 per cent. to invasion by neoplasm. Where it is due to thrombosis this is usually due to spread from veins in the legs and pelvis. In most cases the thrombosis is confined to the lower part of the inferior vena cava, the upper end of the thrombus being below the entrance of the renal veins. It is believed that further extension upwards is prevented by the considerable blood flow from the kidneys. Even if the thrombus extends up to the right auricle it is unusual for the hepatic veins to be obstructed with the development of a BuddChiari syndrome (Harrison et al., 1956).

The following case is of interest because of the extent of the thrombosis and the lack of renal or hepatic symptoms.

\section{Case Report}

M.W.S., a male aged twenty-three, was a naval rating and was well until he was admitted to the Royal Naval Hospital, Bighi, Malta, on August 2 I, 1956, with a pleuritic-like pain in his left chest. There was no relevant past family. or contact history.

On admission his temperature was $99^{\circ}$ and he was found on X-ray to have a small left pleural effusion. His E.S.R. was $51 \mathrm{~mm}$. in $\mathrm{I}$ hour. He was kept at rest and the effusion gradually absorbed. It was presumed to be tuberculous though no bacteriological proof was obtained.

While in bed he first had swelling of his right leg but this was thought to be a transitory phenomenon at that time. He was transferred to the Royal Naval Hospital at Haslar on September I2, 1956. Shortly after admission the left calf became tender and swollen. Thrombosis of a deep vein was diagnosed and the appropriate treatment instigated but this did not include anti-coagulants.
The condition appeared to resolve, but wen the patient began to get up in November ig6, both legs became swollen. There was considerable pitting oedema if he remained on his feetor long. Rest, elastic stockings and exercise weres all tried but with no permanent relief. Superfeial varicosities then appeared in the legs and thighs. He was seen by the surgical specialist on Aprit 5, I957, and he found that the patient had ${ }_{\text {no }}$ evidence of cardiac or renal abnormality, thathis liver and spleen were not enlarged, that there dras no ascites or abdominal mass. The blood pigttre, bleeding and clotting times were normalo जु $\epsilon$ found that both legs became swollen if the stood for any length of time, with pitting oederms spreading up from the ankles. There were prominent subcutaneous veins crossing from thighs to the abdominal wall. It was conside्ced that he had a thrombus at the junction of both common iliac veins and the inferior vena cava $\overrightarrow{\widehat{B}}$ nd that a collateral circulation was forming. Phis opinion was confirmed by the consultant surgen to the Navy, who thought that the block was inot complete, that there was considerable collatgral venous return, and that his oedema would probăbly lessen. He advised that the patient should $n$ tinue with his elastic stockings.

He was invalided from the Navy in June 1957 and was first seen at the Cambridge Chest Clonic in September 1957. His pleural effusion Rad resolved, leaving only a small amount of thickerong at his left costophrenic angle, but he had oedexn? of his legs and there were prominent veins onohis abdominal wall. $\mathrm{He}$ was working as a roglic mechanic, but at the end of the day his legs bectone very swollen and painful.

In February 1958 he was admitted to Addenbrooke's Hospital for a venogram of his infe fior vena cava. It proved impossible to find this femoral veins by percutaneous puncture but this right long saphenous vein was found in the ankle and some phlebograms of his leg were done wipct 


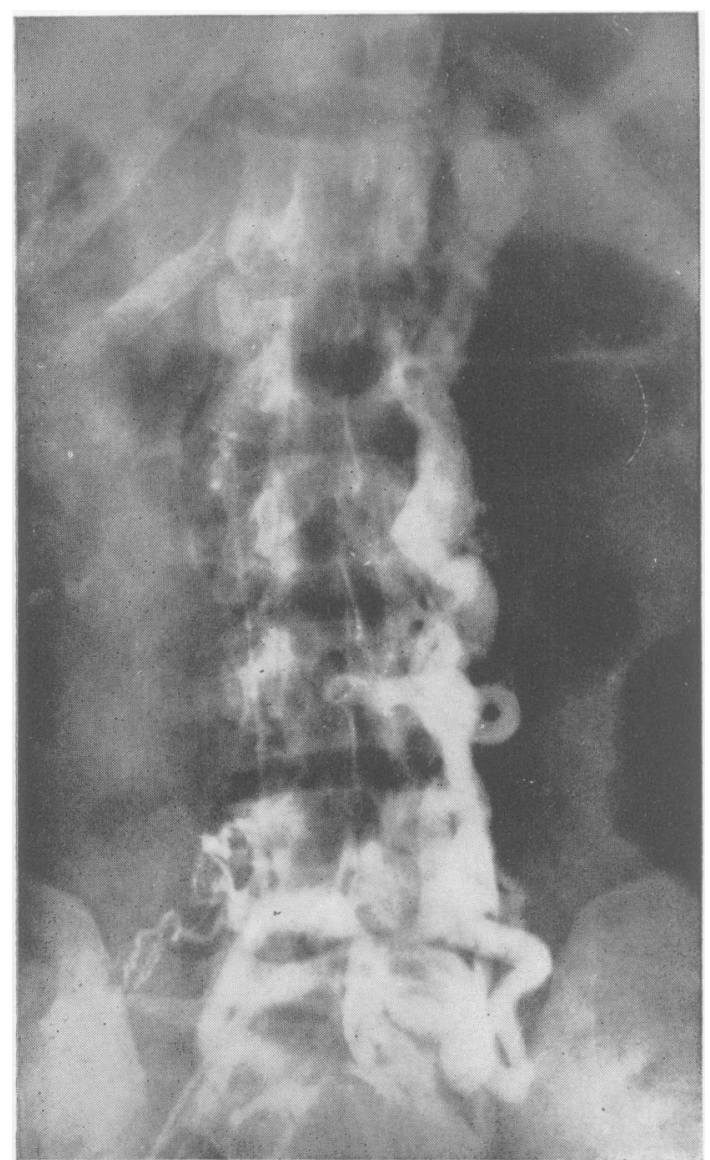

FIG. I

showed that the contrast medium passed into the tibial system of deep veins which appeared segmentally blocked, the saphenous vein also had blocked segments with superficial and deep arastomoses; he did have a deep femoral vein running into his pelvis and this appeared narrowed.

In March I $95^{8}$ another attempt was made to do percutaneous femoral venograms, but this again failed. On the same occasion an attempt was made to do a retrograde inferior vena cavogram by a cardiac catheter passed up through the right basilic vein. However, the catheter would not pass from the right auricle into the inferior vena cava.

In May $195^{8}$ a cut-down dissection was performed on the long saphenous veins in both thighs, polythene catheters were introduced and a venogram performed. This showed that contrast medium passed up the common iliac veins to a point where there was a block at the lower end of the inferior vena cava at the iliac bifurcation.

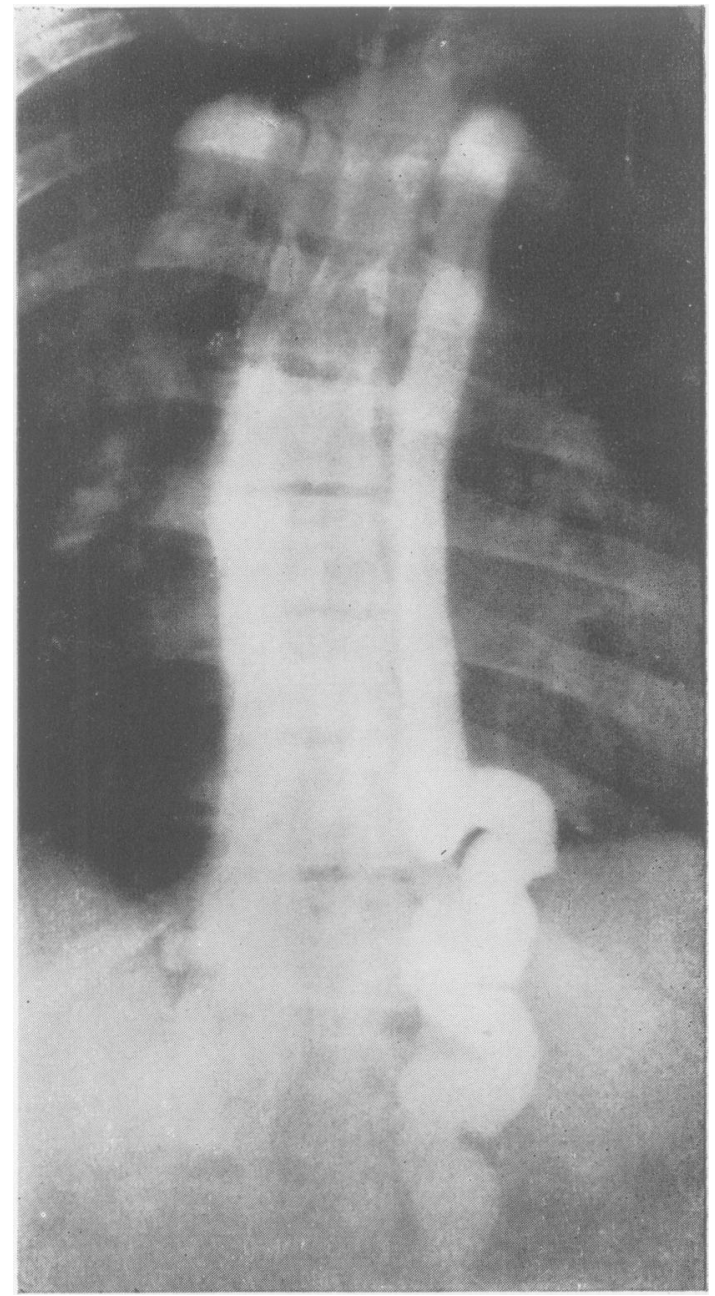

Fig. 2

Gross varicose dilatation of the left ascending lumbar vein and its branches was demonstrated (Fig. I) and the contrast medium filled the ascending lumbar and azygos veins on both sides. Some 'streaking' in the region of the inferior vena cava was noted, which possibly indicated patency of the vasa vasorum. The contrast medium passed up into the thorax via the hemiazygos vein and crossed and filled the azygos vein on the right side. The accessory hemiazygos vein on the left side drained into an intercostal vein. The inferior vena cava itself was never delineated and renal veins were never seen. However, pyelographic excretion into the left kidney was observed. The right auricle opacified but there was no opacification of the upper end of the inferior vena cava suggesting that the block was complete (Fig. 2).

A saphenous vein biopsy was taken which was 


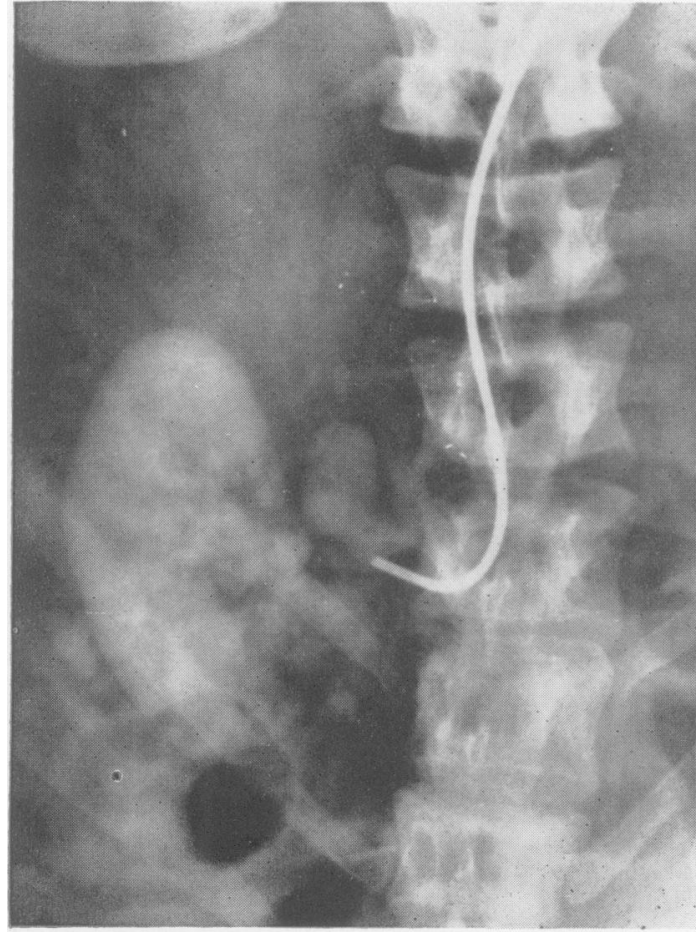

FIG. 3A

histologically normal apart from a small segment of intimal hyperplasia.

In view of the possibility of surgical treatment it was decided to demonstrate the venous drainage of the kidneys. In June $195^{8}$ the left renal artery was first catheterized from the femoral artery and angiography showed arterial filling, renal parenchymal filling and then venous filling. The renal veins drained directly into the lumbar and lumbar azygos veins, i.e. there appeared to be no direct communication from the renal veins to the inferior vena cava (Fig. 3a). A similar state of affairs was found to exist in the right kidney (Fig. 3 b).

Because of the patient's anxiety that something should be done to improve the condition he was referred for further opinion to Professor C. G. Rob, who, however, did not consider that a venous reconstruction procedure would be successful because of the length of the vein graft with consequent high risk of a thrombosis.

The patient is continuing with elastic stockings, sleeping with the foot of the bed raised, and taking chlorothiaziole as a diuretic twice weekly. With this regime his condition has remained stationary. His urine is free of protein and he has had no nephritic symptoms.

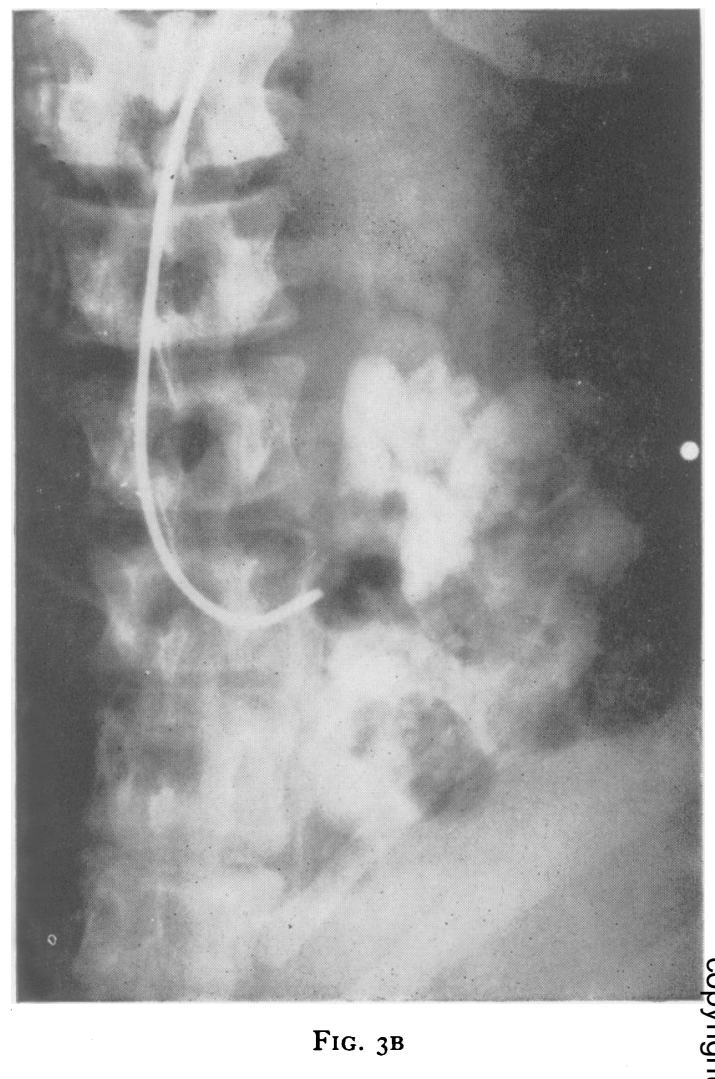

\section{Comment}

In this case the thrombosis probably developed in the veins of the leg while the patient was at rest because of his pleural effusion. There is no evidence of external pressure on the leg veins or inferior vena cava, and from his good general condition it is most unlikely that it could be caused by malignant invasion.

It is perhaps surprising that, despite the extensive thrombosis involving the whole of the inferior vena cava, there was no evidence of renal disfunction, an apparently efficient collateral renal vein drainage having developed. This appears rather similar to the case described by Keen (1940).

\section{Summary}

A case is described of complete obstruction of the inferior vena cava in a young man following a tuberculous pleural effusion. There was no obvious cause. Despite the complete blockage there were no renal or hepatic complications.

\section{BIBLIOGRAPHY}

HARRISON, C. V., MILNE, M. D., and S'TEINER, R. E. (1956) Quart. F. Med., 99, 285.

KEEN, J. A. (1940), Brit. med. F., 2, 823.

PLEASANTS, J. H. (1911), fohns Hopk. Hosp. Rep. 16, 363 